\title{
The Transcriptional Regulator Np20 Is the Zinc Uptake Regulator in Pseudomonas aeruginosa
}

\author{
Matthew L. Ellison ${ }^{1,2^{*}}$, John Matthew Farrow IIII' ${ }^{1}$ Whitney Parrish ${ }^{3}$, Allison S. Danell ${ }^{3}$, Everett C. Pesci ${ }^{1}$ \\ 1 Department of Microbiology and Immunology, East Carolina University, Greenville, North Carolina, United State of America, 2 Department of Biology and \\ Chemistry, Morehead State University, Morehead, Kentucky, United State of America, 3 Department of Chemistry, East Carolina University, Greenville, North \\ Carolina, United States of America.
}

\begin{abstract}
Zinc is essential for all bacteria, but excess amounts of the metal can have toxic effects. To address this, bacteria have developed tightly regulated zinc uptake systems, such as the ZnuABC zinc transporter which is regulated by the Fur-like zinc uptake regulator (Zur). In Pseudomonas aeruginosa, a Zur protein has yet to be identified experimentally, however, sequence alignment revealed that the zinc-responsive transcriptional regulator Np20, encoded by $n p 20$ (PA5499), shares high sequence identity with Zur found in other bacteria. In this study, we set out to determine whether Np20 was functioning as Zur in P. aeruginosa. Using RT-PCR, we determined that np20 (hereafter known as zur) formed a polycistronic operon with znuC and znuB. Mutant strains, lacking the putative $z n u A$, znuB, or znuC genes were found to grow poorly in zinc deplete conditions as compared to wild-type strain PAO1. Intracellular zinc concentrations in strain PAO-Zur ( $\Delta$ zur) were found to be higher than those for strain PAO1, further implicating the zur as the zinc uptake regulator. Reporter gene fusions and real time RT-PCR revealed that transcription of znuA was repressed in a zinc-dependent manner in strain PAO1, however zinc-dependent transcriptional repression was alleviated in strain PAO-Zur, suggesting that the $P$. aeruginosa Zur homolog (ZurPA) directly regulates expression of znuA. Electrophoretic mobility shift assays also revealed that recombinant ZurPA specifically binds to the promoter region of znuA and does not bind in the presence of the zinc chelator $N, N$ ', $N$ tetrakis(2-pyridylmethyl) ethylenediamine (TPEN). Taken together, these data support the notion that Np20 is the $P$. aeruginosa Zur, which regulates the transcription of the genes encoding the high affinity ZnuABC zinc transport system.
\end{abstract}

Citation: Ellison ML, Farrow JM, Parrish W, Danell AS, Pesci EC (2013) The Transcriptional Regulator Np20 Is the Zinc Uptake Regulator in Pseudomonas aeruginosa. PLoS ONE 8(9): e75389. doi:10.1371/journal.pone.0075389

Editor: Shekhar C. Mande, National Centre for Cell Science, India

Received January 4, 2013; Accepted August 15, 2013; Published September 23, 2013

Copyright: @ 2013 Ellison et al. This is an open-access article distributed under the terms of the Creative Commons Attribution License, which permits unrestricted use, distribution, and reproduction in any medium, provided the original author and source are credited.

Funding: This work was supported by a research grant from the National Institute of Allergy and Infectious Disease to ECP (grant AI076272). The funders had no role in study design, data collection and analysis, decision to publish, or preparation of the manuscript.

Competing interests: The authors have declared that no competing interests exist.

*E-mail: M.Ellison@moreheadstate.edu

\section{Introduction}

Zinc is an essential trace element required for virtually all forms of life. The importance of the metal is demonstrated by the fact that $4 \%$ to $10 \%$ of all proteins found throughout the domains of life are zinc metalloproteins [1]. Zinc serves many important functions in such proteins, including acting as a cofactor to facilitate enzymatic reactions, serving as a structural component of many ribosomal proteins and transcriptional regulators, and assisting in proper protein folding and formation. In addition, zinc has been shown to protect against harmful free radical formation which can occur through normal aerobic metabolism within a cell [2]. Accordingly, bacteria concentrate zinc to high levels within the cell to maintain these processes.

In $E$. coli, the intracellular concentration of zinc is approximately $0.2 \mathrm{mM}$, regardless of the zinc concentration in the external milieu [3]. However, even though the intracellular zinc concentrations may be 1000-higher than the extracellular environment, there is typically no free zinc ions found inside the cell [3]. This suggests that the cell possesses means to actively take in the metal and to prevent excess free zinc from accumulating within the bacterium.

Although zinc is essential, excess zinc can have deleterious effects on cells [4]. At physiological concentrations, zinc may have a protective role against free radical formation $[5,6]$. However, surplus zinc has been shown to induce cell toxicity by competing against other metals for protein binding [7] and exposure to high zinc concentrations has been speculated to cause protein denaturation and dysfunction [8]. In addition, zinc induced envelope stress has been shown to disrupt normal physiological processes [9]. Thus, bacteria must not only have the means to take in $\mathrm{Zn}$ (II), but also must repress uptake or quickly export any excess zinc ions to maintain homeostasis. 
To ensure sufficient $\mathrm{Zn}(\mathrm{II})$ is available for cellular functions, many bacteria possess a high affinity active zinc uptake system known as ZnuABC. First identified in Escherichia coli, this ABC-type transport system is comprised of a zinc-binding periplasmic protein (ZnuA), an inner membrane permease (ZnuB), and an ATPase (ZnuC) [10]. In E. coli, Zn(II) uptake by the $Z$ nuABC system is controlled by the zinc uptake regulator (Zur). Zur, a member of the Fur-like family of transcriptional regulators, has the ability to sense and respond to femtomolar changes in zinc concentration within the cell [3]. Zur has at least two zinc binding sites, one zinc binding site ( $C$ site) that allows for structural stability and one or two binding sites ( $M$ and D sites) which regulate DNA binding [11-13]. Under zincreplete conditions, excess zinc ions interact with the $M$ and/or $D$ sites, which causes a conformational change that allows the regulator to bind to the znuA promoter and thereby repress its transcription. In zinc-deplete conditions, the $M$ and/or $D$ sites are unoccupied which leads to destabilization of Zur and the regulator is unable to repress znuA transcription, thus allowing zinc uptake [6].

Our interest in zinc homeostasis, and specifically Zur, stems from recent work with Pseudomonas aeruginosa. $P$. aeruginosa is a ubiquitous gram-negative bacterium found in numerous ecological niches. As an opportunistic pathogen, it can infect a wide range of organisms, including insects, nematodes, plants, and animals [14-18]. In humans, $P$. aeruginosa can cause debilitating disease in the immunocompromised, is a major contributor to nosocomial infections, and is the leading cause of morbidity and mortality in individuals suffering from cystic fibrosis [19]. In order to establish infections, this pathogen produces a host of virulence factors, including the phenazine, pyocyanin. In a study to identify genes involved in the production of pyocyanin, mutation of the $n p 20$ gene which was previously shown to be a virulence determinant [20], caused deficiency in both pyocyanin and the cell-to-cell signal 2-heptyl-3-hydroxy-4-quinolone (the Pseudomonas Quinolone Signal or PQS) production [21]. Others have reported that this regulator is required for production of pyocyanin [22], and that $n p 20$ mutant strains are avirulent in both nematode [15] and mouse infection models [14]. Recently, Np20 was shown to regulate the expression of $d k s A 2$, a paralogue of global transcriptional regulator $d k s A$, in a zinc-dependent manner [23]. The controlled expression of $d k s A 2$ by $\mathrm{Np20}$ allowed $P$. aeruginosa to regulate genes controlled by the zinc-dependent DksA transcriptional regulator in low zinc conditions. Thus, Np20 appears to be involved in the expression of numerous $P$. aeruginosa genes and is responsive to zinc concentration. Despite multiple studies describing its effects, the role of $\mathrm{Np20}$ in regulating zinc homeostasis has not been investigated experimentally. Likewise, no zinc uptake regulator has been identified in $P$. aeruginosa. Based on these observations, we hypothesized that Np20 regulates zinc homeostasis in $P$. aeruginosa. In this study, we present experimental evidence that Np20 serves as a regulator of zinc uptake and hereafter refer to it as $Z_{u_{P A}}$.

\section{Results}

\section{Zur $_{\mathrm{PA}}$ is Highly Similar to Zur Found in Other Bacteria}

If the protein encoded by $n p 20$ is the $P$. aeruginosa Zur, it would be expected to share sequence similarity with Zur proteins found in other bacteria. To address this, we aligned and compared the amino acid sequence of the putative $\operatorname{Zur}_{\mathrm{PA}}$ with that of Zur proteins previously characterized in other bacteria (Figure 1). As can be seen in Figure 1, Zur ${ }_{\mathrm{PA}}$ has high sequence identity with other Zur proteins. It has greater than $40 \%$ identity with the Zur protein from the gram-negative bacteria examined and $45 \%$ similarity ( $31 \%$ identity) with FurB from $M$. tuberculosis (data not shown). Zur $\mathrm{PA}_{\mathrm{PA}}$ also shares several regions of conserved sequences with the other Zur proteins (indicated by the black shading). In addition to containing the first Zur specific sequence, a highly conserved putative DNA-binding region from amino acids 76 to 92, $Z_{\mathrm{ur}_{\mathrm{PA}}}$ also has two conserved zinc binding domains. The double cysteine- $X-X$-cysteine motif found in $C_{118} X X C_{121}-X_{36}-C_{158} X X C_{161}$ is completely conserved in all characterized Zur proteins and is highly conserved throughout the Fur-like protein family [13]. This site corresponds to the structural zinc binding C-site described in other Zur proteins. The other highly conserved site, which includes two histidine residues in the $\mathrm{H}_{109} \mathrm{SH}_{111}$ motif and the highly conserved $\mathrm{C}_{104}$ is indicative of the M-site, which is a zinc sensing site [13]. Zinc interaction with the M-site and portions of the DNA-binding site alters Zur confirmation and allows for DNA binding. The occurrence of the putative $C$ and $\mathrm{M}$ zinc binding sites in $\operatorname{Zur}_{\mathrm{PA}}$ and the high overall similarity between Zur $_{\mathrm{PA}}$ and Zur proteins from other bacteria strongly suggested that the protein encoded by $n p 20$ is the $P$. aeruginosa Zur ( $\left.\mathrm{Zur}_{\mathrm{PA}}\right)$.

\section{zur is in an Operon Which Encodes Components of the Putative ZnuABC System}

We next set out to determine the genetic organization of the operon containing the zur gene. The annotation provided at www.Pseudomonas.com suggested that zur was the first gene in a three gene operon. The genes predicted to be transcribed within the zur operon include homologs of the E. coli znuC $(61 \%$ identity) and znuB $(60 \%$ identity). Using reverse transcription-PCR, we found that primers designed to span the intergenic regions between zur and the znuC homolog, as well as $z n u C$ and $z n u B$ homolog each generated a PCR product (Figure 2A). This indicated that these genes were transcribed on the same mRNA. Interestingly, primers spanning the $42 \mathrm{bp}$ intergenic region between the putative znuB and a hypothetical gene, PA5502, did not give a product, suggesting that gene PA5502 is not part of the zur operon, despite its proximal location to the zur-znuC-znuB operon. As expected, primers spanning the $70 \mathrm{bp}$ intergenic region between zur and the divergently transcribed gene PA5498, which shares $60 \%$ sequence similarity with the $E$. coli znuA (identified as znuA in Figure $2 \mathrm{~A}$ ), did not generate a product. These data suggested that zur forms an operon with two of the three genes which encode components of a probable ZnuABC zinc uptake system, further implicating the involvement of $\operatorname{Zur}_{\mathrm{PA}}$ with the 


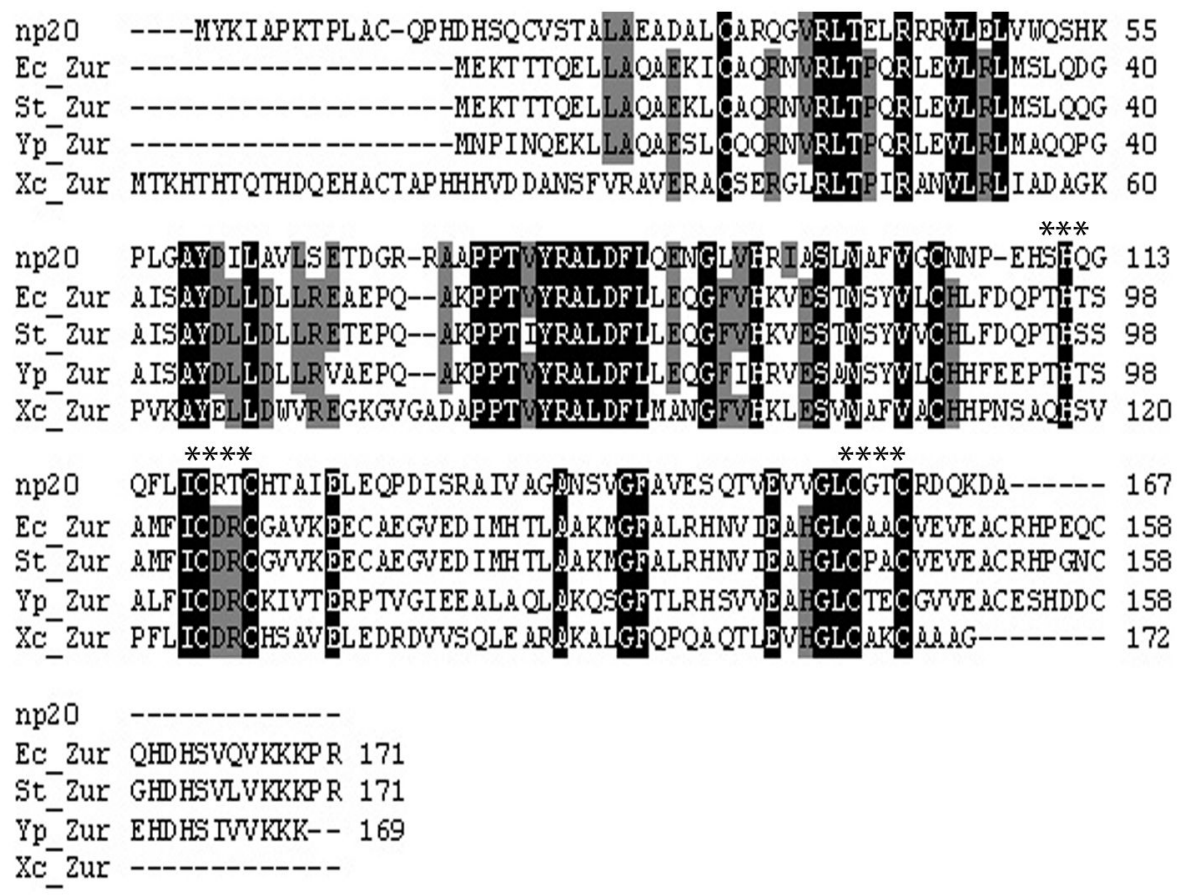

Figure 1. Sequence alignment of $Z_{\mathrm{ur}_{\mathrm{PA}}}$ with other bacterial Zur proteins. The protein sequence of $Z \mathrm{ur}_{\mathrm{PA}}(\mathrm{Np20})$ was aligned with Zur proteins from the following bacteria: E. coli (Ec_Zur); S. typhimurium (St_Zur); Y. pestis (Yp_Zur); X. campestris (Xc_Zur) using ClustalW. Completely conserved residues are indicated by the black shading. Partially conserved residues are indicated by grey shading. Asterisks indicate the $\mathrm{C}$ and $\mathrm{M}$ zinc binding sites.

doi: 10.1371/journal.pone.0075389.g001

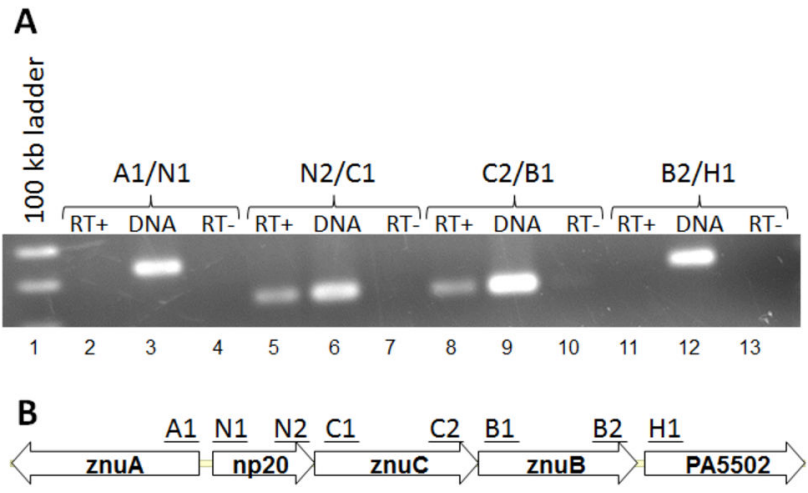

Figure 2. zur is encoded in an operon with znuC and znuB. (A) RT-PCR was performed on total RNA isolated from $P$. aeruginosa strain PA01 using the indicated primer pairs (see Table S2). Reverse Transcriptase was added to the reaction in lanes $2,5,8$, and $11(\mathrm{RT}+)$ but omitted from reactions in lanes $4,7,10$, and 13 (RT-). Genomic DNA was amplified using the indicated primers as a positive control in lanes $3,6,9$, and 12 (DNA); (B) Schematic representation of the zur (np20) operon with relative location of the indicated primers used for RT-PCR. doi: 10.1371/journal.pone.0075389.g002

zinc uptake system. A schematic representation of the Zur operon is shown in Figure 2B.

\section{Loss of the Putative ZnuA, ZnuB, and ZnuC Effects Growth in Low Zinc Conditions}

The similarity of the genes transcribed in the $P$. aeruginosa zur operon to genes in $E$. coli that encode components of the ZnuABC transport system strongly suggest the $P$. aeruginosa homologs would have a similar function. However, many ATPase metal transport systems share high sequence similarities and yet do not transport the same metals. In order to test if the $P$. aeruginosa putative znuA, znuB, and znuC genes may be involved in zinc uptake, we constructed deletion mutants of the putative genes PAO-ZnA $(\Delta z n u A), \mathrm{PAO}-\mathrm{ZnB}$ $(\triangle z n u B)$, and PAO-ZnC $(\Delta z n u C)$, then grew the strains in zinc deplete conditions. As seen in Figure 3 , the inability to express the putative znuA, znuB, or znuC genes resulted in decreased growth at 18 hours as compared wild-type strain PAO1 when the strains were grown in LB media supplemented with $0.5 \mathrm{mM}$ EDTA. The growth defects were not severe $(29.6 \%$ less growth in strain PAO-ZnA, $14.6 \%$ less growth in strain PAO-ZnB, and $14.1 \%$ less growth in strain PAO-ZnC as compared to wild-type strain PAO1), yet they were found to be statistically significant. The ability to grow at all in low zinc can be attributed to $P$. aeruginosa possessing low affinity zinc permeases (such as $\mathrm{HmtA}$ ) that can uptake the metal in the absence of a functional ZnuABC transporter. Because these mutants have decreased growth in low zinc conditions compared to wild-type strain $\mathrm{PAO1}$, and the genes have high sequence similarity to the 


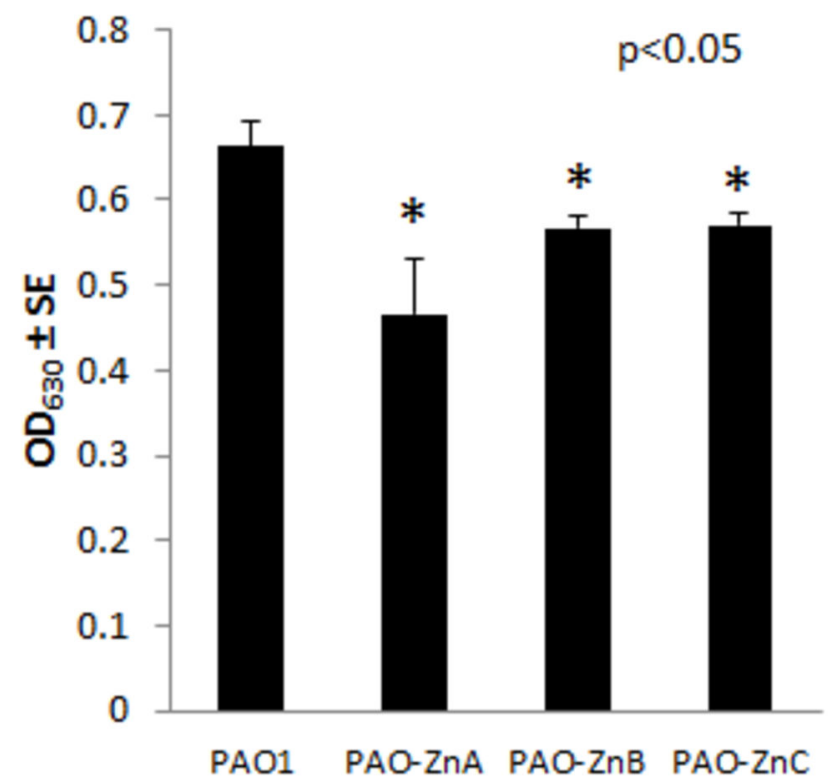

Figure 3. Putative znuA, znuB, and znuC genes are required for wild-type level growth in low zinc conditions. Overnight cultures were diluted in LB media containing $0.5 \mathrm{mM}$ EDTA and grown overnight at $37^{\circ} \mathrm{C}$ for 18 hours with rotary aeration $\geq 220 \mathrm{rpm}$. Growth was assessed spectrophotometrically at absorbance $630 \mathrm{~nm}$. The results are given as the mean of three independent biological replicates. Asterisks indicate statistically significant less growth as compared to wild-type strain PAO1.

doi: 10.1371/journal.pone.0075389.g003

corresponding genes in $E$. coli, these data support the idea that these genes encode the ZnuABC transporter.

To confirm that the mutation of the putative znuA, znuB, or znuC genes did not cause a general growth defect, we analyzed the growth kinetics of strains PAO1, PAO-ZnA, PAO$\mathrm{ZnB}$, and PAO-ZnC grown in LB media (see Figure S1). None of the mutant strains showed growth defects when grown in zinc replete conditions.

\section{Loss of Np20 (Zur) Results in an Accumulation of Intracellular Zinc}

If Np20 is the $P$. aeruginosa Zur, then it should repress zinc uptake in high zinc conditions. Thus, loss of the protein should result in a higher accumulation of intracellular zinc compared to a wild-type strain. To test this hypothesis, we grew strains PAO1 and PAO-Zur in high zinc conditions and then determined the intracellular zinc content by ICP-AES. Our results, shown in Figure 4, show that loss of Zur in strain PAOZur results in a $15.6 \%$ increase of intracellular zinc compared to wild-type strain PAO1 $\left(0.0435 \pm 0.006 \mathrm{mg}\right.$ zinc $/ \mathrm{g}^{-1}$ dry PAOZur cell weight versus $0.0367 \pm 0.002 \mathrm{mg}$ zinc $/ \mathrm{g}^{-1}$ dry PAO1 cell weight). Zinc accumulation inside the PAO-Zur strain did not reach toxic levels, more than likely a result of the activation of zinc efflux systems. However, the results support the notion

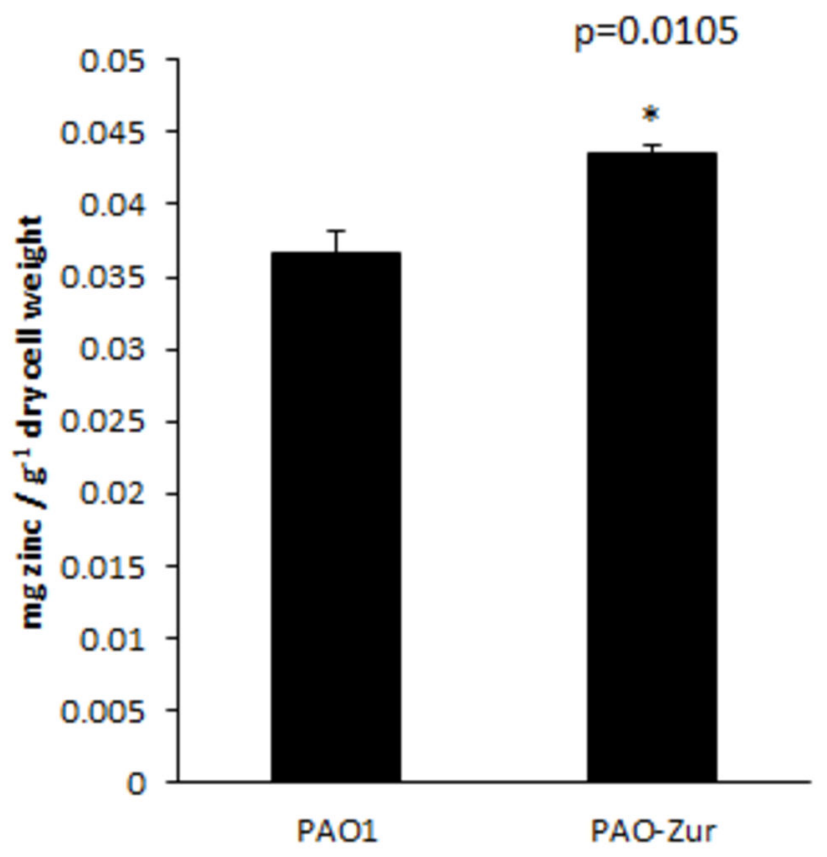

Figure 4. Loss of Zur results in intracellular zinc accumulation. Intracellular zinc content in strains PAO1 and PAO-Zur after growth in high zinc medium as measured by ICP-AES. The data presented are the mean \pm SD of at least four replicates. The asterisk indicates a significant difference between intracellular zinc in stain PAO1 versus PAO-Zur. doi: 10.1371/journal.pone.0075389.g004

that Np20 is the $P$. aeruginosa Zur $\left(\operatorname{Zur}_{\mathrm{PA}}\right)$ and controls zinc uptake in this bacterium.

Transcription of znuA is Under the Control of $\mathrm{Zur}_{\mathrm{PA}}$ in a Zinc-Dependent Manner

To determine the role of $\operatorname{Zur}_{\mathrm{PA}}$ on $z n u A$ transcription, we constructed two reporter strains, PAO1.znuA'-lacZ and PAOZur.znuA'-lacZ, which contain a znuA'-lacZ transcriptional fusion on their chromosomes in a neutral, non-mutational site. The strains were grown to mid-log phase in modified media supplemented with increasing $\mathrm{ZnCl}_{2}$ concentrations, and $\beta$-gal activity was measured. As seen in Figure 5A, znuA transcription was virtually the same for both strains when grown in low zinc media (204 \pm 4 vs. $208 \pm 13$ Miller units). However, the addition of $1 \mu \mathrm{M} \mathrm{ZnCl}$ greatly decreased $z n u A^{\prime}-$ lacZ activity in strain PAO1.znuA'-lacZ to $61 \pm 5$ Miller units while it had virtually no effect on $z n u A^{\prime}-l a c Z$ activity in strain PAO-Zur.znuA'-lacZ (197 \pm 24$)$ (Figure 5A). Transcription of znuA in strain PAO1.znuA'-lacZ decreased step-wise as the $\mathrm{ZnCl}_{2}$ concentrations increased, dropping by greater than 10 fold from M-LB (204 \pm 4 to $18 \pm 4$ Miller units) to M-LB plus 1 $\mathrm{mM} \mathrm{ZnCl}$ (Figure 5A). Transcription of znuA in strain PAOZur.znuA'-lacZ remained relatively unchanged before dropping $35 \%$ when $\mathrm{ZnCl}_{2}$ concentration reached $1 \mathrm{mM}$, indicating that $Z_{\mathrm{ur}}$ is required for a response to zinc. Expression of $Z_{\mathrm{Pu}} \mathrm{PA}_{\mathrm{PA}}$ on plasmid pZur in strain PAO-Zur.znuA'-lacZ when the strain was grown in $\geq 100 \mu \mathrm{M}$ zinc resulted in a drop in Miller units to 15.7 

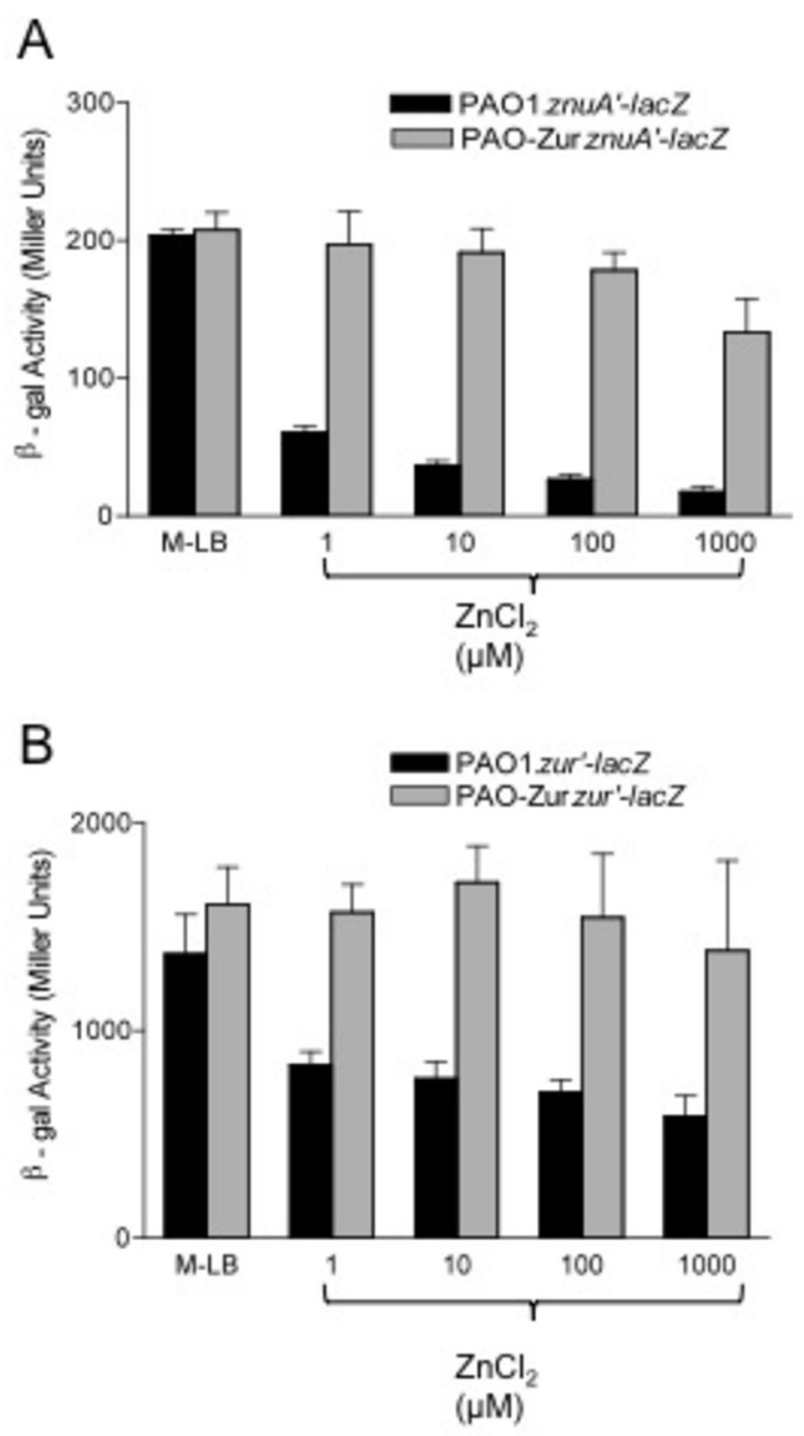

Figure 5. Zur $_{\mathrm{PA}}$ regulates transcription of $z n u A$ and itself. (a) $\beta$-gal assays showing strains PAO1 (black bar) and PAO-Zur ( $\Delta$ zur) (grey bar) containing a chromosomal znuA'lac $Z$ transcriptional fusion grown in increasing concentrations of $\mathrm{ZnCl}_{2}$; (B) $\beta$-gal assays showing strains PAO1(black bar), PAO-Zur ( $\Delta$ zur) and (grey bar) containing a chromosomal zur'lac $Z$ transcriptional fusion grown in increasing concentrations of $\mathrm{ZnCl}_{2}$. The results are presented in Miller Units $\pm \mathrm{SD}$ as the average of at least three independent experiments.

doi: 10.1371/journal.pone.0075389.g005

\pm 7 , which mirrors the Miller Units for strain PAO1.znuA'-lacZ grown under the same conditions (data not shown).

To confirm the $\beta$-galactosidase assay results, we performed real time RT-PCR. We compared expression levels of znuA in strain PAO1 and PAO-Zur grown in M-LB supplemented with 1 $\mathrm{mM} \mathrm{ZnCl}$. Using the house keeping gene rp/U to standardize the comparison, we found that $z n u A$ expression was $5.7 \pm 1.8$ fold higher in strain PAO-Zur than in strain PAO1 when grown in high zinc (data not shown). The fold difference is similar to the 7.5 fold difference in Miller Units between observed between the znuA'-lacZ reporter strains. Collectively, these data support the hypothesis that Zur $_{\mathrm{PA}}$ repressed $z n u A$ transcription in a zinc dependent manner. They also further suggest that $\mathrm{ZnuABC}$ was involved in $\mathrm{Zn}(\mathrm{II})$ homeostasis.

\section{Zur $_{\mathrm{PA}}$ Autoregulates its Own Transcription}

Because the intergenic region between znuA and zur is only $70 \mathrm{bp}$, we speculated that $\operatorname{Zur}_{\mathrm{PA}}$ may also regulate its own transcription. To test this, we constructed a chromosomal zur'lacZ transcriptional fusion reporter, introduced it into a neutral site in strains PAO1 and PAO-Zur, and measured $\beta$ galactosidase activity. As with the $z n u A^{\prime}-l a c Z$ fusion grown in M-LB, transcription of zur in both strains was similar (1368 \pm 191 Miller units vs. $1607 \pm 182$ Miller units; Figure 5B). However, when the strains were grown in the presence of increasing concentrations of $\mathrm{ZnCl}_{2}$, zur transcription was repressed in a step-wise manner in strain PAO1, dropping to 589 \pm 96 Miller units when grown in $1000 \mu \mathrm{M} \mathrm{ZnCl}_{2}$ (Figure 5B). Transcription of zur remained virtually unchanged in strain PAO-Zur.zur'-lacZ regardless of the zinc concentration in the media. Overexpression of Zur $\mathrm{PA}_{\mathrm{PA}}$ on plasmid pZur in PAO-Zur.zur'-lacZ in the presence of excess zinc resulted in almost full complementation of zur repression $(798 \pm 180$ Miller units in PAO-Zur.zur'-lacZ (pZur) versus $705 \pm 58$ Miller units in strain PAO1. zur'-lacZ) when both strains were grown in M-LB plus $100 \mu \mathrm{M} \mathrm{ZnCl}$ (data not shown). These data support the notion that Zur $_{\mathrm{PA}}$ represses transcription of its own operon in response to increases in zinc concentration.

\section{Zur $_{\mathrm{PA}}$ Directly Interacts with the znuA Promoter}

The above data suggested that $Z_{\mathrm{ur}_{\mathrm{PA}}}$ represses znuA transcription in a zinc-dependent manner; however it was not clear if it does so directly. To test whether Zur $_{\mathrm{PA}}$ directly or indirectly regulates the expression of znuA, we performed electrophoretic mobility shift assays (EMSA). Recombinant Strep-tagged $Z_{\mathrm{ur}}$ (rZur) was expressed and purified from $E$. coli strain BL21(DE3) (prZur) and used in EMSA experiments. $P$. aeruginosa rZur bound specifically to the $198 \mathrm{bp}$ fragment which contains the znuA promoter in intergenic region spanning between znuA and zur (Figure 6). The ability of rZur to bind the promoter depended on the availability of free zinc in the buffer reaction. The addition of TPEN, a zinc specific chelator, to the binding reaction rendered $r Z u r$ unable to bind to the promoter. These findings show that $\mathrm{Zn}^{2+}$ can modulate the binding activity of $P$. aeruginosa Zur to DNA and further support the notion that $\operatorname{Zur}_{\mathrm{PA}}$ serves as a zinc-responsive transcriptional repressor of znuA and zur-znuCB.

\section{Discussion}

Most living things require zinc for survival, however excess amounts of this trace element can be toxic. For this reason, most bacteria have developed systems for both zinc acquisition and detoxification. In addition to systems for zinc uptake and efflux, many bacteria have repressors that limit zinc import as the intracellular zinc quota is reached. In this study, we have 


\section{$\operatorname{rZur}(\mu \mathrm{M})$}

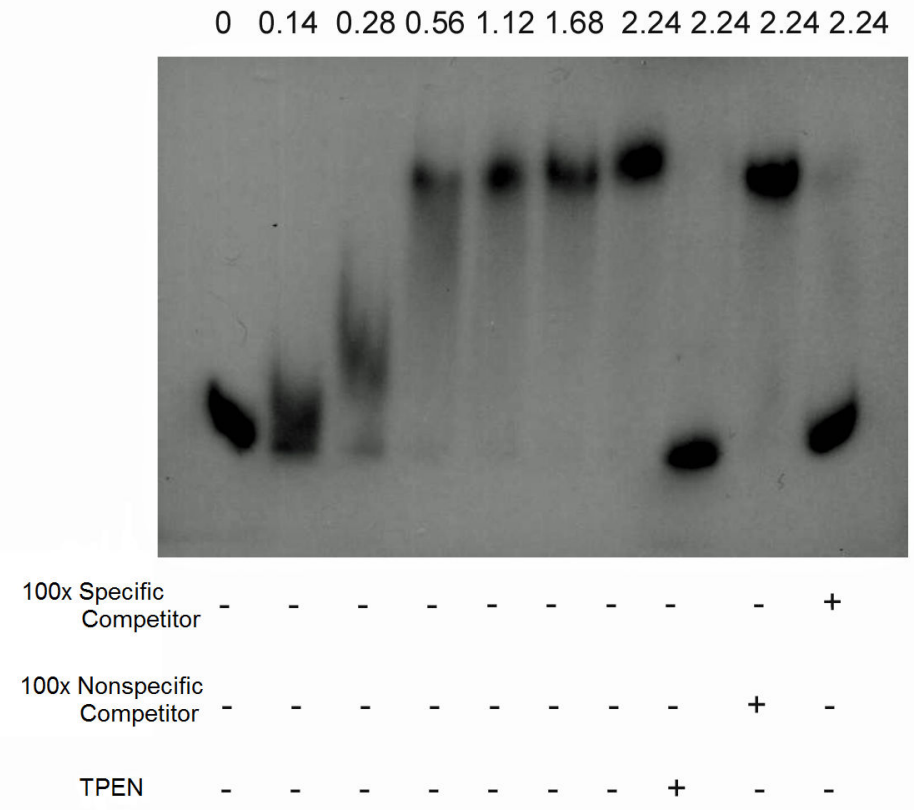

Figure 6. $P$. aeruginosa Zur binds the znuA promoter in a zinc dependent-manner. Radiolabeled znuA promoter fragment was incubated with increasing concentrations of recombinant $P$. aeruginosa Zur ( $r$ Zur) as indicated above the figure. The plus $(+)$ or minus (-) symbols below the lanes indicate whether the reaction contained a 100 -fold excess of unlabeled znuA promoter fragment (specific competitor), unlabeled internal znuC DNA fragment (nonspecific competitor), or $5 \mu \mathrm{M}$ TPEN (zinc chelator).

doi: 10.1371/journal.pone.0075389.g006

presented data which support the notion that the transcriptional regulator encoded by gene $n p 20$ in $P$. aeruginosa acts as a Zur protein by controlling zinc uptake through the zinc specific $A B C$-transport system ZnuABC. Zur ${ }_{P A}$ is zinc responsive and represses transcription of both znuA and the zur-znuC-znuB operon in a zinc dependent manner. Loss of $Z_{u_{P A}}$ results in constitutive transcription of $z n u A$ and zur, regardless of the zinc concentration in the growth media. Loss of Zur also results in the accumulation of intracellular zinc in $P$. aeruginosa. One might expect the loss of Zur to be lethal due to uncontrolled zinc uptake through ZnuABC, but we observed no toxic effects at zinc concentrations at $100 \mu \mathrm{M}$ and only slight effects at extremely high zinc concentrations (data not shown). The reason for this lies in the fact that $P$. aeruginosa possesses several zinc efflux systems. Two such transporters have been characterized in $P$. aeruginosa to date ( $\mathrm{HmtA}$ and $\mathrm{CzcABC})$ and several other potential zinc transport systems exist on the genome $[24,25]$. Our growth data for strain PAO-Zur suggests that loss of Zur does not have deleterious effects as were reported with a CzcA knockout strain (20). Thus, the combined interplay between Zur and zinc efflux systems could explain the minor growth phenotypes observed in strain PAO-Zur. Interestingly, strains $\mathrm{PAO}-\mathrm{ZnA}, \mathrm{PAO}-\mathrm{ZnB}$, and $\mathrm{PAO}-\mathrm{ZnC}$ seemed to grow slightly better than the wild-type strain PAO1 on LB plates supplemented with $10 \mathrm{mM} \mathrm{ZnCl}_{2}$ (data not shown), suggesting that eliminating zinc uptake through ZnuABC offered a protective effect against zinc toxicity. The fact that strains PAO-ZnA, PAO-ZnB, and PAO-ZnC grew well in any media suggests that $P$. aeruginosa can acquire sufficient zinc to sustain life through other means yet to be characterized.

In addition to regulating $Z$ nuABC, studies have begun to expand the Zur regulon far beyond initial expectation. Zur has been shown to regulate a plethora of genes in bacteria, including, zinc acquisition genes [26,27], genes associated with zinc efflux [28], transcriptional regulators [23,29,30], biosynthetic genes responsible for coelibactin production in Streptomyces coelicolor [31], and several ribosomal proteins $[13,20,32,33]$. While the link to genes involved in regulating zinc homeostasis is expected, closer examination of the role that Zur plays in ribosomal protein expression revealed a plausible explanation for a zinc connection. Ribosomal proteins are typically classified into two classes based on their usage of zinc as a cofactor [34]. Zinc-utilizing ribosomal proteins contain two CXXC motifs, similar to the $C$ site found in Zur. Not surprisingly, Zur regulated ribosomal proteins tend to lack zinc binding motifs. Under zinc-replete conditions, Zur represses production of these proteins, leading to preferential production of zinc-utilizing ribosomal proteins. Under zinc-deplete conditions, non-zinc binding proteins make up a majority of the ribosomes [32,34,35]. Thus, Zur can modulate the expression of the proper class of ribosomal proteins and lead to continual protein synthesis in cells in response to zinc availability. In fast growing bacteria, where each cell may contain up to 70,000 
ribosomes [36], this tight regulation is essential to maintain population growth.

The link between Zur and virulence associated transcriptional regulators has sparked a growing interest in recent years. Zur positively regulates the hypersensitivity response and pathogenicity (hrp) transcriptional regulator $h r p X$ in Xanthomonas campestris [29]. HrpX, in turn, regulates expression of the $h r p A$ to $h r p F$, which is required for full virulence in this plant pathogen. A complete Zur - ZnuABC system is required for production of virulence factors and for full virulence in several other pathogens [37-43]. In $P$. aeruginosa, Zur $_{\mathrm{PA}}$ mutants are avirulent in neutropenic mice and nematode infection models and have been reported to produce less PQS [21]. Interestingly, we did not see a difference in $P Q S$ production between the wild-type strain PAO1 or mutant strains PAO-Zur, PAO-ZnA, PAO-ZnB, or PAO-ZnC (data not shown). Loss of znuB caused $P$. aeruginosa to lose cyanide based Caenorhabditis elegans killing [15]. It is somewhat interesting that despite the links to virulence in some bacteria, other pathogens have no requirement for a fully functional Zur - ZnuABC for virulence [44].

Although not as well studied as Fur and iron homeostasis, the expanding knowledge of the Fur-like regulator Zur and the ZnuABC zinc import system suggests that this system is important in numerous physiological processes beyond zinc homeostasis. While parts of the zinc homeostasis system have been identified, the complete system for maintaining the intracellular zinc quota is not well understood. This gap in knowledge along with the link between virulence and zinc homeostasis in $P$. aeruginosa suggests that this field of study shows great promise for helping to understand the role of metals during infection.

\section{Materials and Methods}

\section{Bacterial Strains, Plasmids, and Growth Conditions}

Bacterial strains and plasmids used in this study are listed in Table S1. $P$. aeruginosa strains were maintained at $-70^{\circ} \mathrm{C}$ in $10 \%$ skim milk (Becton Dickinson) and $E$. coli strains were maintained in $15 \%$ glycerol. All cultures were freshly plated for each experiment. Bacteria were cultured in Luria-Bertani (LB) media [45] or zinc deplete modified-LB (M-LB) as noted below. M-LB was prepared by treating LB with Chelex 100 resin (50 $\mathrm{mg} / \mathrm{l}$; Bio-rad, Hercules, Ca) for $1 \mathrm{~h}$ at room temperature to remove heavy metal ions. The resin was removed by filtration and $\quad N, N, N$ ',N'-tetrakis (2-pyridylmethyl)-ethylenediamine (TPEN; Sigma) was added to a final concentration of $20 \mu \mathrm{M}$ to further remove zinc. M-LB was replenished with divalent cations and metals by adding $\mathrm{FeCl}_{3}, \mathrm{CaCl}_{2}$, and $\mathrm{MgCl}_{2}$ to a final concentration of $1 \mu \mathrm{M}, 1 \mu \mathrm{M}$, and $20 \mu \mathrm{M}$, respectively. Finally, $1 \mathrm{ml} / \mathrm{l}$ of trace elements SL-7 solution [46] lacking $\mathrm{Zn}$ was added. When necessary, cultures were supplemented with 200 $\mu \mathrm{g} / \mathrm{ml}$ carbenicillin, $100 \mu \mathrm{g} / \mathrm{ml}$ ampicillin, $30 \mu \mathrm{g} / \mathrm{ml}$ gentamicin, or $50 \mu \mathrm{g} / \mathrm{ml}$ kanamycin to maintain plasmids.

\section{Generation of Mutant Strains}

Unmarked in-frame deletion mutagenesis was used to create mutants by allelic exchange using a modification [47] of the previously described method by Horton et al. (1989) [48]. Briefly, mutant alleles were constructed using a two-step PCR protocol to create in-frame deletions of the DNA coding sequence corresponding to amino acids 28 to 138 (72\%) of zur (PA5499), 39 to 285 (80\%) of znuA (PA5498), 40 to 223 (80\%) of znuB (PA5500), and 38 to $226(70 \%)$ of znuC (PA5501). The oligonucleotide primers used to produce the mutant alleles added an Xbal site to the ends of the mutant zur allele, EcoRI sites to the end of $z n u A$, and Hindlll sites to the ends of $z n u B$, and znuC mutant alleles. The DNA fragments were digested with the appropriate enzyme, purified from an agarose gel, and ligated into $\mathrm{pEX} 18 \mathrm{Ap}$, which had been previously digested with the same enzyme. This produced suicide plasmids pZur-suc, pZnuA-suc, pZnuB-suc, and pZnuC-suc.

To introduce the mutant alleles into $P$. aeruginosa strain PAO1, this strain was transformed with each suicide vector using the electroporation method described by Choi and Schweizer [49]. Single cross-over integrants were selected on carbenicillin and screened for sucrose sensitivity. Mutants were then selected by plating integrants on LB plus $6 \%$ sucrose to remove the vector from the chromosome. The resultant colonies were screened on LB containing carbenicillin to ensure carbenicillin resistance had been lost. Potential mutants were then screened by PCR and confirmed by DNA sequencing.

\section{Construction of lacZ Reporter Plasmids and $\beta$ - Galactosidase ( $\beta-\mathrm{Gal}$ ) Assays}

The chromosomal znuA'-lacZ transcriptional fusion reporter strains $P$. aeruginosa PAO1.znuA'-lac $Z$ and $P$. aeruginosa PAO-Zur.znuA'-lacZ were constructed using the method described by Choi and Schweizer [50]. A 425 bp product beginning $387 \mathrm{bp}$ upstream from the znuA translational start site was amplified by PCR using strain PAO1 chromosomal DNA as template and primers designed to add a Pstl site at the 5 ' and an HindIII site at the 3' end. The fragment was digested with Pstl and HindIII, purified from an agarose gel, and ligated into the pUC18-mini-TnTT-Gm-lacZ plasmid, which had been previously digested with the same restriction enzymes. The resultant plasmid, pznuA'-lacZ was sequenced to confirm no mutations were introduced during cloning. The $z n u A^{\prime}-l a c Z$ fusion was inserted into the attTn7 site on the $P$. aeruginosa strain PAO1 and strain PAO-Zur chromosomes as described elsewhere [49]. The gentamicin resistance cassette was excised using the pFLP2-encoded Flp recombinase, and the pFLP2 plasmid was cured via plating on $6 \%$ sucrose. A zur'lacZ reporter plasmid was constructed by amplifying a $464 \mathrm{bp}$ sequence containing the zur promoter $(-307$ to +164 relative to the zur translational start sight) by PCR using cosmid pMTP331 as a template. The primers were designed to add a Pstl site at the 5' end of the PCR product and an HindIII site at the 3' end. The PCR fragment was digested with Pstl and HindIII, purified from an agarose gel, and ligated into pUC18mini-Tn $7 T-G m-l a c Z$ plasmid which had been previously digested with the same enzymes to produce pzur'-lacZ. 
Plasmids were sequenced to ensure that mutations were not introduced by PCR manipulations and the plasmids were introduced into the att $T n 7$ site on the $P$. aeruginosa strain PAO1 and PAO-Zur by electroporation as described above [49]. The gentamicin resistance cassette was excised from the chromosome using pFLP2 as described elsewhere [50].

To determine the effect of different zinc concentrations on znuA expression, cells from an overnight culture of $P$. aeruginosa strain PAO1.znuA'-lacZ and PAO-Zur.znuA'-lacZ were washed once in $M-L B$ and used to inoculate $1 \mathrm{ml}$ of fresh M-LB or M-LB, supplemented with different $\mathrm{ZnCl}_{2}$ concentrations, to an optical density at $660 \mathrm{~nm}\left(\mathrm{OD}_{660}\right)$ of 0.08 . The cultures were grown for $3 \mathrm{~h}$ at $37^{\circ} \mathrm{C}$ with rotary aeration of $\geq 200 \mathrm{rpm}$ and the $\beta$-Gal activity of each sample was assayed in duplicate. In some instances, PAO-Zur.znuA'-lacZ was transformed with pZur and $\beta$-gal assays were performed in $\mathrm{M}$ LB supplemented with $\mathrm{ZnCl}_{2}$, carbenicillin $200 \mu \mathrm{g} / \mathrm{ml}$, and $1 \%$ arabinose as indicated.

Data are reported in Miller units as the mean \pm standard deviation (SD) of at least three separate experiments.

To determine the role of Zur on zur expression, cells from an overnight culture of $P$. aeruginosa strain PAO1.zur'-lacZ and PAO-Zur.zur'-lacZ were washed once in M-LB and used to inoculate $1 \mathrm{ml}$ of fresh M-LB or M-LB, supplemented with different $\mathrm{ZnCl}_{2}$ concentrations, to an optical density $660 \mathrm{~nm}$ $\left(O D_{660}\right)$ of 0.08 . The cultures were grown for $3 \mathrm{~h}$ at $37^{\circ} \mathrm{C}$ with rotary aeration of $\geq 200 \mathrm{rpm}$ and the $\beta-G a l$ activity of each sample was assayed in duplicate. Data are reported in Miller units as the mean \pm standard deviation of at least three separate experiments.

\section{Construction of a Zur ${ }_{\mathrm{PA}}$ Expression Plasmid}

To construct a $Z_{\mathrm{PA}} \mathrm{PA}_{\mathrm{P}}$ complementation plasmid, the oligonucleotide primers Zur_F and Zur_R (see Table S2) were used to amplify a $505 \mathrm{bp}$ fragment containing the zur coding fragment from cosmid MTP331. The primers were designed to add a Pcil site to the 5' end and a Hindlll site to the 3' end of the PCR product. The PCR product was purified from an agarose gel, digested with $\mathrm{Ncol}$ and HindlII, and ligated into pHerd20T which had been digested with the same enzymes. The resulting plasmid, pZur, was sequenced to confirm that no mutations were introduced during DNA manipulation.

\section{Bioinformatics}

The protein sequence of previously characterized Zur proteins found in Escherichia coli, Salmonella typhimurium, Yersinia pestis, and Xanthomonas campestris were obtained from the NCBI database. The protein sequence alignment of select Zur proteins was generated using ClustalW [51].

\section{RT-PCR}

Total RNA was prepared from $P$. aeruginosa strain PAO1 mid-log phase cultures grown in LB using a Qiagen RNase Mini Kit. Contaminating DNA was removed by digestion with $10 \mathrm{U}$ of $\mathrm{RQ1}$ RNase-free DNase (Promega) for $2 \mathrm{~h}$ at $37^{\circ} \mathrm{C}$. DNA-free total RNA was then used as a template for reverse transcriptase reactions using the Access RT-PCR Introductory System (Promega). The primers used in the RT-PCR reactions are listed in Table $\mathrm{S} 2$ and were in the following sets: $\mathrm{A} 1, \mathrm{~N} 1$; N2, C1; C2, B1; B2, H1.

\section{Growth Kinetic Bioassays}

The ability of strains PAO1, PAO-ZnA, PAO-ZnB, and PAO$\mathrm{ZnC}$ to grow in zinc deplete conditions was determined by growth bioassays. Briefly, overnight cultures were diluted in LB supplemented with $0.5 \mathrm{mM}$ EDTA to an $\mathrm{OD}_{630}$ of approximately 0.025 . The cultures were grown at $37^{\circ} \mathrm{C}$ for $18 \mathrm{~h}$ with rotary aeration of $\geq 220 \mathrm{rpm}$. Growth was determined spectrophotometrically and results are presented as the mean of three independent biological replicates. Statistical significance of growth patterns was determined by the student T-test.

\section{Determination of Intracellular Zinc Concentrations}

The intracellular concentration of zinc inside strain PAO1 and strain PAO-Zur was determined by inductively coupled plasma atomic emission spectroscopy (ICP-AES). Overnight cultures of strain PAO1 and PAO-ZnC were washed in LB and used to inoculate $5 \mathrm{ml} L B$ to an $\mathrm{OD}_{660}$ of 0.05 . The cultures were grown at $37^{\circ} \mathrm{C}$ with rotary aeration of $\geq 220 \mathrm{rpm}$ for $2.5 \mathrm{~h}$, then $\mathrm{ZnCl}_{2}$ was added to final concentration of $100 \mu \mathrm{M}$ and the cultures were incubates as before for $3 \mathrm{~h}$. The cells were pelleted by centrifugation at $5,000 \times \mathrm{g}$ for $5 \mathrm{~min}$. at $4^{\circ} \mathrm{C}$ and then washed sequentially in $10 \mathrm{ml} \mathrm{LB}$ and $10 \mathrm{ml}$ wash buffer $(0.1 \mathrm{M}$ lithium chloride, $0.2 \mathrm{mM}$ ethylenediaminetetraacetic acid, and $0.1 \mathrm{mM}$ ethylene glycol tetraacetic acid) to remove exogenous zinc. The cells were placed at $80^{\circ} \mathrm{C}$ overnight to dehydrate the cells, their dry weight was determined, and then incinerated at $>800^{\circ} \mathrm{C}$ overnight. The samples were reconstituted in $2 \%$ nitric acid to a mass of $10 \mathrm{~g}$. The total zinc content was determined using (ICP-AES). The results of at least four replicates are reported as total zinc $(\mathrm{mg})$ per gram of dry bacterial cell weight.

\section{Real Time RT-PCR}

Total RNA was isolated from strains PAO1 and PAO-Zur grown in M-LB supplemented with $1 \mathrm{mM} \mathrm{ZnCl}_{2}$ to mid-log phase using a RNeasy Midi Kit (Qiagen) according to the manufacturer's instructions. Contaminating DNA was removed using DNA-free (Ambion) Dnase Treatment and cDNA was generated using $2 \mu \mathrm{g}$ total RNA, random primers, and Superscript III (Invitrogen) per manufacturer's suggestions. The resultant cDNA was diluted 1:100 in nuclease free water and stored at $-20^{\circ} \mathrm{C}$ until needed. Real time RT-PCR reactions were performed on a CFX96 real time system (Biorad) using $0.5 \mu \mathrm{l}$ dilute cDNA as template, $200 \mathrm{nM}$ final concentration of primers ZnuA_rt1 and ZnuA_rt2, or primer rplU_rt1 and rplU_rt2, $7.5 \mu \mathrm{l}$ of PerfeCTa SYPR Green FastMix 2X mix (Quanta Biosciences), and ultrapure water up to a final volume of $25 \mu \mathrm{l}$. The ratio of znuA expression in strain PAO1 compared to strain PAO-Zur grown in high zinc concentrations was determined using the Pfaffl equation [52] using expression of $r p / U$ as a constant. The ratio was determined from the averages of at least three replicates from three independent biological replicates. 


\section{Expression and Purification of Recombinant Zur $_{\mathrm{PA}}$}

To construct a plasmid for expression of recombinant Zur ${ }_{\mathrm{PA}}$, the oligonucleotide primers rZur_F and rZur_R (see Table S2) were used to amplify a $536 \mathrm{bp}$ fragment containing the zur coding region with Bsal restriction sites added to the $5^{\prime}$ and $3^{\prime}$ ends. The PCR product was gel purified, digested with Bsal, and ligated into pASK-IBA6 (IBA GMH, Göttingen, Germany), which had been previously digested with the same enzyme. The resulting plasmid, prZur, containing a N-terminal Strep-tag II version of $P$. aeruginosa Zur ( (Zur $\left._{\mathrm{PA}}\right)$, was introduced into $E$. coli strain $\mathrm{DH} 5 \mathrm{a}$ by chemical transformation. A high concentration of prZur was purified from E. coli strain DH5a using a Qiagen Plasmid Midi kit, the plasmid was sequenced to insure no mutations were introduced during cloning, and the plasmid was transformed into chemically competent $E$. coli BL21(DE3).

For expression and purification of the $\mathrm{rZur}_{\mathrm{PA}}$, overnight $E$. coli BL21(DE3) (prZur) cultures were used to inoculate $1 \mathrm{~L}$ of LB supplemented with ampicillin $(100 \mu \mathrm{g} / \mathrm{ml})$ contained in a $4 \mathrm{~L}$ growth flask. The cultures were grown at $37^{\circ} \mathrm{C}$ with rotary aeration $\geq 280 \mathrm{rpm}$ until the $\mathrm{OD}_{590}$ reached 0.6 , at which time anhydrotetracycline was added to a final concentration of 0.2 $\mu \mathrm{g} / \mathrm{ml}$ and the cultures were incubated at $30^{\circ} \mathrm{C}$ with rotary aeration $\geq 280 \mathrm{rpm}$ for $3 \mathrm{~h}$. All subsequent steps were performed on ice or at $4^{\circ} \mathrm{C}$. Cells were harvested by centrifugation at $5,000 \times \mathrm{g}$ for $5 \mathrm{~min}$, and the cells were suspended in $10 \mathrm{ml}$ lysis buffer $(10 \mathrm{mM}$ Tris- $\mathrm{HCl}, \mathrm{pH} 8.0,40$ $\mathrm{mM} \mathrm{KCl}, 10 \mathrm{mM} \mathrm{MgCl}$, $1 \mathrm{mM} \mathrm{DTT}$, and $5 \%$ glycerol). Then the cells were lysed by sonication using a Branson Sonifer 450 ( $7 \mathrm{x}$ $20 \mathrm{sec}$ ). The lysate was centrifuged at $5,000 \times \mathrm{g}$ for $15 \mathrm{~min}$ to remove the insoluble fraction and the aqueous lysate was passed through a $0.22 \mu \mathrm{M}$ syringe filter apparatus. The lysate was applied to Strep-tactin gravity flow column (IBA GMH) per manufacturer's instructions. Protein fractions were pooled and dialyzed in storage buffer (10 mM Tris- $\mathrm{HCl}, \mathrm{pH} 8.0,40 \mathrm{mM} \mathrm{KCl}$, $10 \mathrm{mM} \mathrm{MgCl}_{2}, 1 \mathrm{mM} \mathrm{DTT}$, and $10 \%$ glycerol) and concentrated using an Amicon Ultra centrifugal filter. The protein concentration was determined by Bradford assays and the protein was stored at $-70^{\circ} \mathrm{C}$ until used.

\section{Electrophoretic Mobility Shift Assays (EMSA)}

The ability of $\operatorname{Zur}_{P A}$ to bind the $z n u A$ promoter was determined by EMSA essentially as described by Knoten et al. [53] Briefly, a 259 bp PCR fragment containing the znuA promoter (227 bp upstream from the GTG start codon to $32 \mathrm{bp}$ downstream) was generated using strain PAO1 chromosomal DNA as a template and was labeled with [32P]ATP (PerkinElmer, Wellesley, MA) by using T4 polynucleotide

\section{References}

1. Andreini C, Banci L, Bertini I, Rosato A (2006) Counting the zincproteins encoded in the human genome. J Proteome Res 5: 196-201. doi:10.1021/pr050361j. PubMed: 16396512.

2. Coudray C, Rachidi S, Favier A (1993) Effect of zinc on superoxidedependent hydroxyl radical production in vitro. Biol Trace Elem Res 38: 273-287. doi:10.1007/BF02785311. PubMed: 7504944

3. Outten CE, O'Halloran TV (2001) Femtomolar sensitivity of metalloregulatory proteins controlling zinc homeostasis. Science 292: 2488-2492. doi:10.1126/science.1060331. PubMed: 11397910. kinase (Invitrogen, Carlsbad, CA). The binding assays were carried out in binding buffer containing $10 \mathrm{mM}$ Tris- $\mathrm{HCl}, \mathrm{pH} 8.0$, $40 \mathrm{mM} \mathrm{KCl}, 10 \mathrm{mM} \mathrm{MgCl}_{2}, 1 \mathrm{mM} \mathrm{DTT}$, and $5 \%$ glycerol and 1 $\mu \mathrm{M} \mathrm{ZnCl}$. Each reaction mixture contained $0.5 \mu \mathrm{g}$ of salmon sperm DNA, $10^{4} \mathrm{cpm}$ radiolabeled DNA, and 0 to $800 \mathrm{ng}$ of protein. Where indicated, 100x excess specific competitor (unlabeled znuA promoter), 100x excess non-specific competitor (unlabeled internal fragment of znuC), or $5 \mu \mathrm{M}$ TPEN was added to the binding reaction. Reaction mixtures were incubated at room temperature for $30 \mathrm{~min}$ and separated by electrophoresis at $4{ }^{\circ} \mathrm{C}$ on a native $7.5 \%$ polyacrylamide gel supplemented with $2.5 \%$ glycerol in $1 \mathrm{X}$ Tris-borate buffer. Radiolabeled bands were visualized by autoradiography after the X-ray film had been exposed for $18 \mathrm{~h}$.

\section{Supporting Information}

Figure S1. Growth Kinetics of $P$. aeruginosa strains PAO1, PAO-ZnA, PAO-ZnB, and PAO-ZnC grown in LB medium. Overnight cultures of each strain were diluted in fresh LB media to an $\mathrm{OD}_{630}$ of 0.005 and the cultures were incubated at $37^{\circ} \mathrm{C}$ with rotary aeration $\geq 220 \mathrm{rpm}$. Growth was monitored spectrophotometrically for 6 hours. The results presented are the mean of three independent experiments.

(TIF)

Table S1. List of bacterial strains and plasmids used in this study.

(DOCX)

Table S2. List of primers used in this study. (DOCX)

\section{Acknowledgements}

The authors would like to thank William G. Wells, Kyle A. Tipton, and Claire A. Knoten for critically reviewing this paper. We would also like to thank Metrics, Inc. (Greenville, NC) for allowing access to their ICP-AES. We would also like to thank Dr. Nathan Coker for technical assistance with the ICP-AES experiments.

\section{Author Contributions}

Conceived and designed the experiments: MLE ASD ECP. Performed the experiments: MLE JMF WP. Analyzed the data: MLE ECP. Contributed reagents/materials/analysis tools: ASD ECP. Wrote the manuscript: MLE JMF ECP.

4. Silver S (1996) Bacterial resistances to toxic metal ions--a review. Gene 179: 9-19. doi:10.1016/S0378-1119(96)00323-X. PubMed: 8991852.

5. Bray TM, Bettger WJ (1990) The physiological role of zinc as an antioxidant. Free Radic Biol Med 8: 281-291. doi: 10.1016/0891-5849(90)90076-U. PubMed: 2187766

6. Smith KF, Bibb LA, Schmitt MP, Oram DM (2009) Regulation and activity of a zinc uptake regulator, Zur, in Corynebacterium diphtheriae. 
J Bacteriol 191: 1595-1603. doi:10.1128/JB.01392-08. PubMed: 19074382.

7. McDevitt CA, Ogunniyi AD, Valkov E, Lawrence MC, Kobe B et al. (2011) A molecular mechanism for bacterial susceptibility to zinc. PLOS Pathog 7: e1002357.

8. Yamamoto K, Ishihama A (2005) Transcriptional response of Escherichia coli to external zinc. J Bacteriol 187: 6333-6340. doi: 10.1128/JB.187.18.6333-6340.2005. PubMed: 16159766.

9. Mellies JL, Thomas K, Turvey M, Evans NR, Crane J et al. (2012) Zincinduced envelope stress diminishes type III secretion in enteropathogenic Escherichia coli. BMC Microbiol 12: 123. doi: 10.1186/1471-2180-12-123. PubMed: 22727253.

10. Patzer SI, Hantke K (1998) The ZnuABC high-affinity zinc uptake system and its regulator Zur in Escherichia coli. Mol Microbiol 28: 1199-1210. doi:10.1046/j.1365-2958.1998.00883.x. PubMed: 9680209.

11. Lucarelli D, Russo S, Garman E, Milano A, Meyer-Klaucke W et al. (2007) Crystal structure and function of the zinc uptake regulator FurB from Mycobacterium tuberculosis. J Biol Chem 282: 9914-9922. doi: 10.1074/jbc.M609974200. PubMed: 17213192.

12. Outten CE, Tobin DA, Penner-Hahn JE, O'Halloran TV (2001) Characterization of the metal receptor sites in Escherichia coli Zur, an ultrasensitive zinc(II) metalloregulatory protein. Biochemistry 40: 10417-10423. doi:10.1021/bi0155448. PubMed: 11523983.

13. Shin JH, Jung HJ, An YJ, Cho YB, Cha SS et al. (2011) Graded expression of zinc-responsive genes through two regulatory zincbinding sites in Zur. Proc Natl Acad Sci U S A 108: 5045-5050. doi: 10.1073/pnas.1017744108. PubMed: 21383173

14. Cao H, Baldini RL, Rahme LG (2001) Common mechanisms for pathogens of plants and animals. Annu Rev Phytopathol 39: 259-284. doi:10.1146/annurev. phyto.39.1.259. PubMed: 11701866.

15. Gallagher LA, Manoil C (2001) Pseudomonas aeruginosa PAO1 kills Caenorhabditis elegans by cyanide poisoning. J Bacteriol 183: 6207-6214. doi:10.1128/JB.183.21.6207-6214.2001. PubMed: 11591663.

16. Lau GW, Ran H, Kong F, Hassett DJ, Mavrodi D (2004) Pseudomonas aeruginosa pyocyanin is critical for lung infection in mice. Infect Immun 72: 4275-4278. doi:10.1128/IAl.72.7.4275-4278.2004. PubMed: 15213173.

17. Mahajan-Miklos S, Tan MW, Rahme LG, Ausubel FM (1999) Molecular mechanisms of bacterial virulence elucidated using a Pseudomonas aeruginosa-Caenorhabditis elegans pathogenesis model. Cell 96: 47-56. doi:10.1016/S0092-8674(00)80958-7. PubMed: 9989496.

18. Rahme LG, Tan MW, Le L, Wong SM, Tompkins RG et al. (1997) Use of model plant hosts to identify Pseudomonas aeruginosa virulence factors. Proc Natl Acad Sci U S A 94: 13245-13250. doi:10.1073/pnas. 94.24.13245. PubMed: 9371831.

19. Khan TZ, Wagener JS, Bost T, Martinez J, Accurso FJ et al. (1995) Early pulmonary inflammation in infants with cystic fibrosis. Am J Respir Crit Care Med 151: 1075-1082. doi:10.1164/ajrccm/151.4.1075. PubMed: 7697234

20. Li Y, Qiu Y, Gao H, Guo Z, Han Y et al. (2009) Characterization of Zurdependent genes and direct Zur targets in Yersinia pestis. BMC Microbiol 9: 128. doi:10.1186/1471-2180-9-128. PubMed: 19552825

21. Gallagher LA, McKnight SL, Kuznetsova MS, Pesci EC, Manoil C (2002) Functions required for extracellular quinolone signaling by Pseudomonas aeruginosa. J Bacteriol 184: 6472-6480. doi:10.1128/JB. 184.23.6472-6480.2002. PubMed: 12426334.

22. Dietrich LE, Price-Whelan A, Petersen A, Whiteley M, Newman DK (2006) The phenazine pyocyanin is a terminal signalling factor in the quorum sensing network of Pseudomonas aeruginosa. Mol Microbiol 61: 1308-1321. doi:10.1111/j.1365-2958.2006.05306.x. PubMed: 16879411.

23. Blaby-Haas CE, Furman R, Rodionov DA, Artsimovitch I, de CrécyLagard V (2011) Role of a Zn-independent DksA in Zn homeostasis and stringent response. Mol Microbiol 79: 700-715. doi:10.1111/j. 1365-2958.2010.07475.x. PubMed: 21255113.

24. Lewinson O, Lee AT, Rees DC (2009) A P-type ATPase importer that discriminates between essential and toxic transition metals. Proc Natl Acad Sci U S A 106: 4677-4682. doi:10.1073/pnas.0900666106. PubMed: 19264958

25. Hassan MT, van der Lelie D, Springael D, Römling $U$, Ahmed $N$ et al. (1999) Identification of a gene cluster, czr, involved in cadmium and zinc resistance in Pseudomonas aeruginosa. Gene 238: 417-425. doi: 10.1016/S0378-1119(99)00349-2. PubMed: 10570969.

26. Petrarca P, Ammendola S, Pasquali P, Battistoni A (2010) The Zurregulated ZinT protein is an auxiliary component of the high-affinity ZnuABC zinc transporter that facilitates metal recruitment during severe zinc shortage. J Bacteriol 192: 1553-1564. doi:10.1128/JB.01310-09. PubMed: 20097857.
27. Stork M, Bos MP, Jongerius I, de Kok N, Schilders I et al. (2010) An outer membrane receptor of Neisseria meningitidis involved in zinc acquisition with vaccine potential. PLOS Pathog 6: e1000969. PubMed: 20617164.

28. Huang DL, Tang DJ, Liao Q, Li HC, Chen Q et al. (2008) The Zur of Xanthomonas campestris functions as a repressor and an activator of putative zinc homeostasis genes via recognizing two distinct sequences within its target promoters. Nucleic Acids Res 36: 4295-4309. doi:10.1093/nar/gkn328. PubMed: 18586823.

29. Huang DL, Tang DJ, Liao Q, Li XQ, He YQ et al. (2009) The Zur of Xanthomonas campestris is involved in hypersensitive response and positively regulates the expression of the hrp cluster via hrpX but not hrpG. Mol Plant Microbe Interact 22: 321-329. doi:10.1094/ MPMI-22-3-0321. PubMed: 19245326.

30. Maciag A, Dainese E, Rodriguez GM, Milano A, Provvedi $R$ et al. (2007) Global analysis of the Mycobacterium tuberculosis Zur (FurB) regulon. J Bacteriol 189: 730-740. doi:10.1128/JB.01190-06. PubMed: 17098899.

31. Kallifidas D, Pascoe B, Owen GA, Strain-Damerell CM, Hong HJ et al. (2010) The zinc-responsive regulator Zur controls expression of the coelibactin gene cluster in Streptomyces coelicolor. J Bacteriol 192: 608-611. doi:10.1128/JB.01022-09. PubMed: 19915027.

32. Gabriel SE, Helmann JD (2009) Contributions of Zur-controlled ribosomal proteins to growth under zinc starvation conditions. J Bacteriol 191: 6116-6122. doi:10.1128/JB.00802-09. PubMed: 19648245.

33. Gabriel SE, Miyagi F, Gaballa A, Helmann JD (2008) Regulation of the Bacillus subtilis yciC gene and insights into the DNA-binding specificity of the zinc-sensing metalloregulator Zur. J Bacteriol 190: 3482-3488. doi:10.1128/JB.01978-07. PubMed: 18344368.

34. Makarova KS, Ponomarev VA, Koonin EV (2001) Two C or not two C: recurrent disruption of $\mathrm{Zn}$-ribbons, gene duplication, lineage-specific gene loss, and horizontal gene transfer in evolution of bacterial ribosomal proteins. Genome Biol 2: RESEARCH 0033

35. Panina EM, Mironov AA, Gelfand MS (2003) Comparative genomics of bacterial zinc regulons: enhanced ion transport, pathogenesis, and rearrangement of ribosomal proteins. Proc Natl Acad Sci U S A 100: 9912-9917. doi:10.1073/pnas.1733691100. PubMed: 12904577.

36. Bremer H, Dennis PP (1996) Modulation of chemical composition and other parameters of the cell by growth rate. In: FC Neidhardt; Rci JL.Ingraham ECC, Lin KB, Low B, Magasanik WS, Rezinkoff M et al., Editors. Escherichia coli and Salmonella: cellular and molecular biology, 2nd ed. Washington, D.C.: ASM Press. pp. 1553-1569

37. Ammendola $S$, Pasquali $P$, Pistoia $C$, Petrucci $P$, Petrarca $P$ et al. (2007) High-affinity Zn2+ uptake system ZnuABC is required for bacterial zinc homeostasis in intracellular environments and contributes to the virulence of Salmonella enterica. Infect Immun 75: 5867-5876. doi:10.1128/IAI.00559-07. PubMed: 17923515.

38. Campoy S, Jara M, Busquets N, Pérez De Rozas AM, Badiola I et al. (2002) Role of the high-affinity zinc uptake znuABC system in Salmonella enterica serovar typhimurium virulence. Infect Immun 70: 4721-4725. doi:10.1128/IAl.70.8.4721-4725.2002. PubMed: 12117991.

39. Davis LM, Kakuda T, DiRita VJ (2009) A Campylobacter jejuni znuA orthologue is essential for growth in low-zinc environments and chick colonization. J Bacteriol 191: 1631-1640. doi:10.1128/JB.01394-08. PubMed: 19103921.

40. Kim S, Watanabe K, Shirahata T, Watarai M (2004) Zinc uptake system (znuA locus) of Brucella abortus is essential for intracellular survival and virulence in mice. J Vet Med Sci 66: 1059-1063. doi:10.1292/jvms. 66.1059. PubMed: 15472468

41. Lewis DA, Klesney-Tait J, Lumbley SR, Ward CK, Latimer JL et al. (1999) Identification of the znuA-encoded periplasmic zinc transport protein of Haemophilus ducreyi. Infect Immun 67: 5060-5068. PubMed: 10496878

42. Sabri M, Houle S, Dozois CM (2009) Roles of the extraintestinal pathogenic Escherichia coli ZnuACB and ZupT zinc transporters during urinary tract infection. Infect Immun 77: 1155-1164. doi:10.1128/IAl. 01082-08. PubMed: 19103764.

43. Weston BF, Brenot A, Caparon MG (2009) The metal homeostasis protein, Lsp, of Streptococcus pyogenes is necessary for acquisition of zinc and virulence. Infect Immun 77: 2840-2848. doi:10.1128/IAI. 01299-08. PubMed: 19398546.

44. Desrosiers DC, Bearden SW, Mier I Jr., Abney J, Paulley JT et al. (2010) Znu is the predominant zinc importer in Yersinia pestis during in vitro growth but is not essential for virulence. Infect Immun 78: 5163-5177. doi:10.1128/IAI.00732-10. PubMed: 20855510.

45. Sambrook J, Russell DW (2001) Molecular clonging: a laboratory manual. Cold Spring Harbor, NY: Cold Spring Harbor Laboratory Press. 
46. Atlas RM (2010) Handbook of Microbiological Media, 4th Edition. Boca Raton, FL: CRC Press.

47. Farrow JM 3rd, Sund ZM, Ellison ML, Wade DS, Coleman JP et al. (2008) PqsE functions independently of PqsR-Pseudomonas quinolone signal and enhances the rhl quorum-sensing system. J Bacteriol 190: 7043-7051. doi:10.1128/JB.00753-08. PubMed: 18776012.

48. Horton RM, Hunt HD, Ho SN, Pullen JK, Pease LR (1989) Engineering hybrid genes without the use of restriction enzymes: gene splicing by overlap extension. Gene 77: 61-68. doi: 10.1016/0378-1119(89)90359-4. PubMed: 2744488.

49. Choi KH, Kumar A, Schweizer HP (2006) A 10-min method for preparation of highly electrocompetent Pseudomonas aeruginosa cells: application for DNA fragment transfer between chromosomes and plasmid transformation. J Microbiol Methods 64: 391-397. doi:10.1016/ j.mimet.2005.06.001. PubMed: 15987659.

50. Choi KH, Schweizer HP (2006) mini-Tn7 insertion in bacteria with single attTn7 sites: example Pseudomonas aeruginosa. Nat Protoc 1: 153-161. doi:10.1038/nprot.2006.24. PubMed: 17406227.

51. Chenna R, Sugawara H, Koike T, Lopez R, Gibson TJ et al. (2003) Multiple sequence alignment with the Clustal series of programs. Nucleic Acids Res 31: 3497-3500. doi:10.1093/nar/gkg500. PubMed: 12824352.

52. Pfaffl MW (2001) A new mathematical model for relative quantification in real-time RT-PCR. Nucleic Acids Res 29: e45. doi:10.1093/nar/ 29.9.e45. PubMed: 11328886.

53. Knoten CA, Hudson LL, Coleman JP, Farrow JM 3rd, Pesci EC (2011) $\mathrm{KynR}$, a Lrp/AsnC-type transcriptional regulator, directly controls the kynurenine pathway in Pseudomonas aeruginosa. J Bacteriol 193: 6567-6575. doi:10.1128/JB.05803-11. PubMed: 21965577. 